\title{
Predictors of Scalp Hair Thinning in Women with Polycystic Ovary Syndrome
}

\author{
Deborah E Ikhena, ${ }^{1}$ Joanne Williams, ${ }^{2}$ Nighat Naqvi ${ }^{2}$ and Lubna Pal ${ }^{2}$
}

1. Department of Obstetrics and Gynecology, Division of Reproductive Endocrinology and Infertility, Northwestern University Feinberg School of Medicine, Chicago, US; 2. Department of Obstetrics, Gynecology and Reproductive Sciences, Yale University School of Medicine, New Haven, US

\begin{abstract}
Objective: To determine the prevalence and predictors of perceived scalp hair thinning (SHT) among women with polycystic ovary syndrome (PCOS). Materials and Methods: A cross-sectional study of women with PCOS to determine the prevalence and predictors of SHT in this population. The sample included 263 women presenting to a reproductive endocrinologist at a tertiary care teaching institution for symptoms relating to PCOS. The main outcome measures were symptoms of PCOS including SHT, quality of life, hormonal and metabolic parameters. Clinical, hormonal and metabolic predictors of SHT were determined utilizing multivariable logistic regression analyses. Results: SHT was acknowledged by $27 \%$ of subjects. Advancing age ( $\mathrm{p}=0.024)$, subjective hirsutism (odds ratio [OR] 2.0, 95\% $\mathrm{Cl} 1.1-3.91$ ), disturbed sleep (OR $1.91,95 \% \mathrm{Cl} 1.1-3.4$ ) and smoking (OR 1.90, 95\% Cl 1.0-3.9) were associated with significantly increased likelihood of $\mathrm{SHT}$ ( $\mathrm{p}<0.05)$. Serum androgen levels did not relate to SHT. On analyses adjusting for age, body mass index, race, and smoking status, abnormal two-hour oral glucose tolerance test (OR 9.9, 95\% Cl 3.1-30.2), serum low-density lipoprotein (LDL) (OR 0.96, 95\% Cl 0.97-0.99), and skipped meals (OR 3.1, $95 \% \mathrm{Cl}$ 1.3-7.3) were independent predictors of SHT in women with PCOS. Conclusions: Similar to acanthosis nigricans, our data identify SHT as a phenotypic reflection of concomitant metabolic burden in women with PCOS.
\end{abstract}

\section{Keywords}

PCOS, Hair loss, scalp hair thinning, androgens, glucose, androgenetic alopecia

Disclosure: Deborah E Ikhena, Joanne Williams and Nighat Naqvi have nothing to disclose in relation to this article. Lubna Pal is a consultant for Merck and Co. No funding was received in relation to the publication of this article.

Compliance with Ethics: Patient data were retrospectively evaluated for this cross-sectional study. Institutional approval was obtained and given the design, the study was deemed exempt from need to procure subject consent. All procedures were followed in accordance with the responsible committee on human experimentation and with the Helsinki Declaration of 1975 and subsequent revisions.

open Access: This article is published under the Creative Commons Attribution Noncommercial License, which permits any non-commercial use, distribution, adaptation and reproduction provided the original author(s) and source are given appropriate credit.

Received: January 16, 2016 Accepted: February 23, 2016 Citation: US Endocrinology 2016;12(1):26-30 DOI: http://doi.org/10.17925/USE.2016.12.01.26

Correspondence: Lubna Pal, Department of Obstetrics, Gynecology and Reproductive Sciences, Yale University School of Medicine, 333 Cedar Street, PO Box 208063,

New Haven, CT 06510, US. E: lubna.pal@yale.edu

Polycystic ovary syndrome (PCOS) is the most common endocrine disorder in reproductive age women. $^{1}$ According to National Institutes of Health $(\mathrm{NIH})$ criteria (presence of ovulatory dysfunction and hyperandrogenism, after exclusion of related disorders), PCOS is estimated to affect $6-8 \%$ of women in the US, the prevalence is estimated to be almost doubled to $15 \%$ when on the Rotterdam criteria are utilized. ${ }^{2,3}$

Common PCOS-related symptoms include excess facial and body hair, acne, and irregular menses secondary to anovulation. ${ }^{1}$ Androgenetic alopecia (AA), or female pattern hair loss is also a recognized symptom of PCOS. It is a non-scarring form of alopecia that is characterized by scalp hair thinning (SHT). This is more pronounced in the vertex of the scalp, with preservation of the frontal hairline. ${ }^{4}$ It affects $6 \%$ of women under the age of $50,{ }^{4}$ although detection rates of $22-35 \%$ have been noted in women presenting for evaluation for hyperandrogenism. ${ }^{5-8}$
The underlying mechanisms of SHT in AA are follicular miniaturization and decreased length of the anagen (growth) phase of the hair cycle. ${ }^{4,9}$ A familial pattern of inheritance of AA has also been suggested. ${ }^{10}$

The prevalence of depressive symptoms is disproportionately higher in the PCOS population compared with age-matched healthy women. ${ }^{11}$ While the exact causes of mood disturbances in PCOS are unclear, phenotypic abnormalities (hirsutism, acne, obesity) as well as the endocrine milieu (androgen and insulin excess) have been variously suggested as being contributory. Independent of PCOS diagnosis, AA imposes a significant psychological burden on women. Perceptions of feeling "less attractive and ashamed" and reporting of depressive symptoms secondary to an altered body image are well described in association with AA. ${ }^{8}$ Indeed, women with perceived SHT experience more negative psychological effects than their male counterparts. ${ }^{12}$ 
The objective of the study was to determine the prevalence of subjective SHT in women with PCOS and to define the predictors of subjective SHT in this population of women.

\section{Materials and methods Subjects}

Subjects were women of reproductive age, referred to a reproductive endocrinologist at a tertiary care teaching institution for symptoms relating to PCOS (menstrual abnormalities, hair excess, acne, or biochemical evidence of hyperandrogenemia) from January 2009 and October 2012. Patient data were retrospectively evaluated for this cross-sectional study. Institutional approval was obtained and given the design, the study was deemed exempt from need to procure subject consent.

Diagnosis of PCOS was based on the Rotterdam criteria, ${ }^{3}$ after exclusion of common endocrine disorders that can mimic PCOS such as thyroid function, hyperprolactinemia, and adrenal disorders. ${ }^{2}$ subjects were excluded if they had been on any hormonal therapy for PCOS in the three months prior to presentation. Subjects were evaluated as per routine clinical practice with detailed intake of medical, social, and family histories. Oligomenorrhea was defined as menstrual cycle length greater than 35 days. ${ }^{13}$ History of depression, anxiety (Yes/ No), subjective perception of SHT (Yes/No), smoking (Yes/No), regular recreational exercise (Yes/No), disturbances in nocturnal sleep (Yes/ No) and skipped meals (Yes/No) were obtained. Subjects completed a validated nine-item personal health questionnaire (PHQ), which aimed to identify depressive symptoms based on PHQ scores. ${ }^{14}$ Presence and severity of hair excess was assessed through administration of modified Ferriman-Gallwey (mFG) pictorial chart that subjects self-scored; $\mathrm{mFG}$ score greater than eight was deemed to reflect hirsutism. ${ }^{15}$ Body mass index (BMI) was calculated (weight in kilograms divided by height in meters ${ }^{2}$ ). Subjects on any form of hormonal therapy were excluded from this analysis.

\section{Laboratory analysis}

Biochemical and hormonal tests were performed at local clinical laboratories. Hormonal and metabolic evaluation included assessment of serum total testosterone ( $T, n g / d L, n=222)$, dehydroepiandrosterone sulfate (DHEAS, $n g / d L, n=211$ ), sex hormone binding globulin (SHBG, $n m o l / L, n=190)$, free testosterone ( $\mathrm{pg} / \mathrm{dL}, \mathrm{n}=209)$, fasting glucose $(\mathrm{mg} / \mathrm{dL}$, $n=227)$, fasting insulin ( $\mu \mathrm{IU} / \mathrm{ML}, \mathrm{n}=184)$, and fasting lipid profile $(n=200)$. Total testosterone was measured in serum using turbulent flow liquid chromatography tandem mass spectrometry. A fasting two-hour oral 75 g glucose tolerance test (OGTT) was obtained in a subset $(n=206)$ and plasma glucose and insulin levels were measured at 0, 30, 60, 90, and 120 minutes. Abnormalities in glucose homeostasis included impaired fasting glucose (IFG, defined as fasting plasma glucose between 100 and 125 mg/ $\mathrm{dL}$ ), impaired glucose tolerance (IGT, defined as a plasma glucose value between 144 and $199 \mathrm{mg} / \mathrm{dL}$ at 120 minutes after a $75 \mathrm{~g}$ oral glucose load) and diabetes mellitus (DM, defined as plasma glucose greater than $200 \mathrm{mg} / \mathrm{dL}$ two hours after a $75 \mathrm{~g}$ oral glucose load, or a fasting glucose level greater than or equal to $126 \mathrm{mg} / \mathrm{dL}$ ). ${ }^{16}$ All subjects with IGT or DM were labeled as having abnormal glucose homeostasis. Homeostatic model assessment of insulin resistance (HOMA-IR) was calculated by multiplying fasting insulin ( $\mu \mathrm{IU} / \mathrm{ml}$ ) by fasting glucose $\mathrm{dg} / \mathrm{ml}$ and dividing the product by $405 .{ }^{17}$

\section{Table 1: Demographic and clinical characteristics of women with PCOS by presence or absence of scalp hair thinning}

\begin{tabular}{llll} 
Parameter & $\begin{array}{l}\text { Reporting SHT } \\
(\mathrm{n}=70)\end{array}$ & $\begin{array}{l}\text { Without SHT } \\
(\mathrm{n}=193)\end{array}$ & P value \\
\hline Age (years) & $29 \pm 6.3$ & $27 \pm 6.2$ & 0.024 \\
\hline BMI (kg/m²) & $33.8 \pm 10.5$ & $33.1 \pm 8.3$ & 0.578 \\
\hline Smoking (\%) & $25 \%$ & $14 \%$ & 0.048 \\
\hline Alcohol (\%) & $23 \%$ & $20 \%$ & 0.552 \\
\hline Race & & & 0.002 \\
\hline - White & $45(66 \%)$ & $127(67 \%)$ & \\
\hline - Black & $3(4 \%)$ & $33(17 \%)$ & 0.05 \\
\hline - Asian & $12(18 \%)$ & $10(5 \%)$ & 0.02 \\
\hline - Other & $8(12 \%)$ & $21(11 \%)$ & 0.45 \\
\hline Oligomenorrhea (\%) & $80 \%$ & $86 \%$ & 0.229 \\
\hline Subjective hirsutism (\%) & $80 \%$ & $66 \%$ & 0.032 \\
\hline Acne (\%) & $56 \%$ & $55 \%$ & 0.909 \\
\hline Disturbed sleep & $43 \%$ & $29 \%$ & 0.025 \\
\hline Skipped meals (\%) & $46 \%$ & $27 \%$ & 0.007 \\
\hline Depression (\%) & $33 \%$ & $32 \%$ & 0.789 \\
\hline Anxiety (\%) & $35 \%$ & $26 \%$ & 0.196 \\
\hline Exercise (\%) & $48 \%$ & $44 \%$ & 0.639 \\
\hline Systolic BP (mm Hg) & $114.7 \pm 17$ & $114.6 \pm 14$ & 0.985 \\
\hline Diastolic BP (mm Hg) & $71 \pm 10$ & $71 \pm 10$ & 0.838 \\
\hline Objective hirsutism & $47 \%$ & $48 \%$ & 0.976 \\
\hline Conth & & &
\end{tabular}

Continuous data are presented as mean (SD) and categorical as percentage.

Subjective reporting of excess facial and body hair on modified Ferriman-Gallwey scale; $M F G>8 . B M l=$ body mass index; $B P=$ blood pressure; $P C O S=$ polycystic ovary syndrome; $S H T=$ scalp hair thinning

\section{Statistical analysis}

The primary outcome was the presence or absence of SHT and analyses explored predictors of SHT in this population. Continuous variables were analyzed using parametric (Pearson's correlation and Student's T test) or non-parametric (Spearman correlation, Mann-Whitney $U$ test) measures as appropriate. Categorical data were compared using chi-square test. Tertiles for the individual biomarkers were computed and Kruskall Wallis Rank test compared distribution of biomarker tertiles by presence or absence of SHT.

Multivariable logistic regression analysis determined predictors of SHT (dependent variable) in women with PCOS. Model diagnostics assessed the goodness of model fit. Continuous data demonstrating Gaussian distribution are presented as mean \pm standard deviation (SD) and skewed data are presented as median and interquartile range (IQR). Categorical data are presented as percentage. Associations are presented as odds ratio $(O R) \pm 95 \%$ confidence interval $(95 \% \mathrm{Cl})$. STATA 12.0 was used for analyses and $p<0.05$ was considered statistically significant.

\section{Results \\ Demographics}

A total of 263 women were diagnosed with PCOS based on the Rotterdam criteria and were included in the study. Of these, 222 (84\%) had clinical or biochemical evidence of hyperandrogenism and 195 (74\%) met the NIH diagnostic criteria for PCOS diagnosis. Twenty-seven percent of subjects $(n=70)$ reported SHT. Demographic and clinical characteristics of subjects with versus without SHT are presented in Table 1. Women with SHT were, on average, older than those without SHT $(p=0.024)$. Racial differences in the prevalence of SHT were apparent; Asian women with PCOS were 


\section{Table 2: Endocrine and metabolic parameters in women with and without complaint of scalp hair thinning}

\begin{tabular}{|c|c|c|c|}
\hline & $\begin{array}{l}\text { Reporting SHT } \\
(n=70)\end{array}$ & $\begin{array}{l}\text { Without SHT } \\
(n=193)\end{array}$ & $P$ value \\
\hline $\left.\operatorname{DHEAS}^{\mathrm{a}}(\mu \mathrm{g} / \mathrm{dL})\right)$ & $213.9 \pm 150.6$ & $214.5 \pm 125.2$ & 0.556 \\
\hline Total Testosterone $(\mathrm{ng} / \mathrm{dL})$ & $40.2 \pm 26.9$ & $40.3 \pm 27.4$ & 0.759 \\
\hline Free Testosterone $^{c}$ & $3.4(1.7-5.9)$ & $4(1.9-7)$ & 0.190 \\
\hline $\mathrm{SHBG}^{\mathrm{d}}(\mathrm{nmol} / \mathrm{L})$ & $38(26-74)$ & $33(22-57)$ & 0.134 \\
\hline Fasting Glucose $\mathrm{e}^{\mathrm{mg} / \mathrm{dl})}$ & $93 \pm 18$ & $88 \pm 16$ & 0.029 \\
\hline Fasting Insulinf (ulU/ml) & $10(5-18)$ & $9(5-16)$ & 0.472 \\
\hline HOMA-IR & $1.8(0.8-3.5)$ & $1.8(0.8-2.8)$ & 0.570 \\
\hline Abnormal 2h OGTTh(\%) & $27 \%$ & $10 \%$ & 0.002 \\
\hline Total Cholesterol (mg/dl) & $169 \pm 36.1$ & $175 \pm 38.0$ & 0.321 \\
\hline LDL cholesterol (mg/dl) & $88 \pm 34.6$ & $105 \pm 32.4$ & 0.002 \\
\hline HDL cholesterol (mg/dl) & $53 \pm 18.7$ & $53 \pm 23.7$ & 0.957 \\
\hline HDH:LDL Ratioi & $0.57(0.40-0.87)$ & $0.49(0.36-0.67)$ & 0.045 \\
\hline Triglycerides (mg/dl) & $119 \pm 76.1$ & $112 \pm 71.4$ & 0.556 \\
\hline
\end{tabular}

Normally distributed continuous data are presented as mean (SD) and skewed data as median (IQR) Categorical data presented as percentage. ${ }^{a}$ DHEAS levels were available in 57 with SHT and 154 without SHT; ${ }^{b}$ Total testosterone available in 64/70 with SHT and 158/193 without SHT. ' Free testosterone levels available in 61/70 with SHT and 148/193 without SHT; median (IQR); 'dSHBG levels available in 55/70 with SHT and 135/193 without SHT; median (IQR); ${ }^{e}$ fasting glucose levels available in 61/70 with SHT and 166/193 without SHT; ${ }^{f}$ fasting insulin levels available in 54/70 with SHT and 130/193 without SHT; gHOMA-IR available in 54/70 with SHT and 130/193 without SHT; median (IOR);

"Abnormal 2 h OGTT was undertaken in 51/70 with SHT and 155/193 without SHT; abnormal OGTT included evidence of either IGT or DM based on specified thresholds of glucose levels, ${ }^{i}$ HDL:LDL ratio: Levels available in 52 with SHT and 141 without SHT; $H E A S=$ dehydroepiandrosterone sulfate: $H D L=$ high-density lipoprotein:

HOMA-IR= Homeostatic model assessment of insulin resistance; $S H B G=$ sex hormone binding globulin; SHT= scalp hair thinning

more likely to acknowledge SHT $(p=0.020)$ compared with Caucasian women (Table 1). Those who reported skipped meals were significantly more likely to acknowledge SHT ( $p=0.007)$ (Table 1). Subjective hirsutism ( $p=0.032)$, disturbed sleep $(p=0.025)$, and smoking $(p=0.048)$ were positively associated with SHT (Table 1). The presence of menstrual irregularity and acne, as well as BMI was comparable between those with and without subjective SHT (Table 1).

\section{Endocrine and metabolic parameters}

Serum androgen levels (total and free testosterone and DHEAS) did not relate to the symptom of SHT (Table 2). Fasting insulin and HOMA-IR levels were comparable between the two groups (Table 2). Fasting glucose was significantly higher in women who reported SHT $(p=0.029)$. Although women with SHT had significantly higher plasma glucose levels at 60 , 90 , and 120 minutes following a $75 \mathrm{~g}$ oral glucose load (Figure 1), insulin levels during the course of the OGTT were comparable between the two groups (Figure 2). An abnormal two-hour oral glucose tolerance test, defined as a plasma glucose value greater than $144 \mathrm{mg} / \mathrm{dL}$ following a 75 g oral glucose load (IGT or DM) was associated with a threefold increased likelihood of SHT compared with women with a normal glucose profile (OR 3.5, 95\% Cl 1.5-8.2, p=0.003) (Table 3). Conversely, women reporting SHT had a significantly higher prevalence of IFG, IGT and DM $(p=0.054)$ (Figure 3). There was no difference in total cholesterol, high-density lipoprotein (HDL) or triglyceride levels between groups. However, women who reported SHT had lower low-density lipoprotein (LDL) levels (Table 2). Serum SHBG levels were observed to be higher in women reporting SHT compared with those without this symptom, although this difference was not of statistical significance on univariate analyses (Table 2).

\section{Figure 1: Glucose response to $75 \mathrm{gm}$ Oral Glucose $(n=193)$}

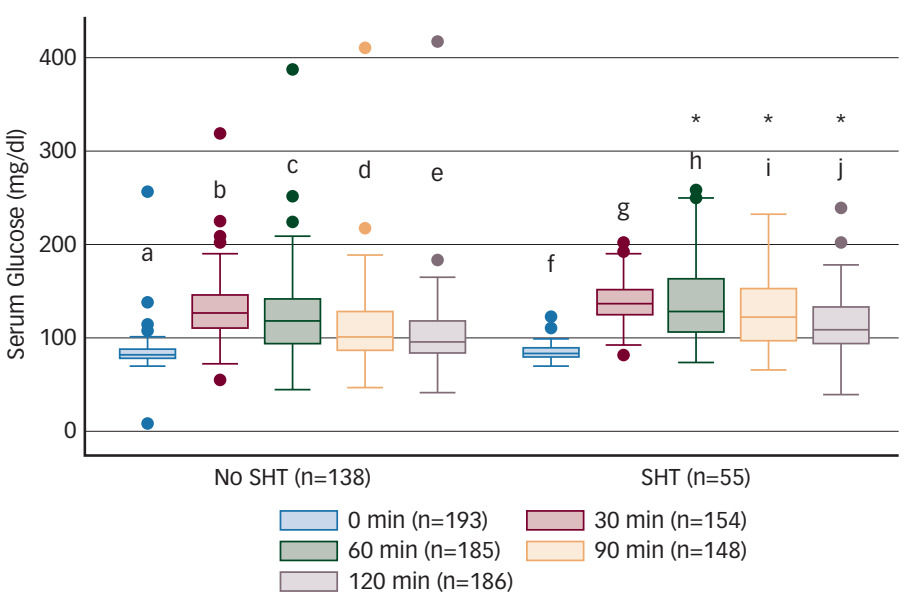

a versus $f, p=0.96 ; b$ versus $g, p=0.38 ; c$ versus $h, p=0.02 ; d$ versus $i, p=0.02$; e versus $j$, $p=0.04$. SHT $=$ scalp hair thinning

\section{Figure 2: Insulin response to $75 \mathrm{gm}$ oral glucose $(n=184)$}

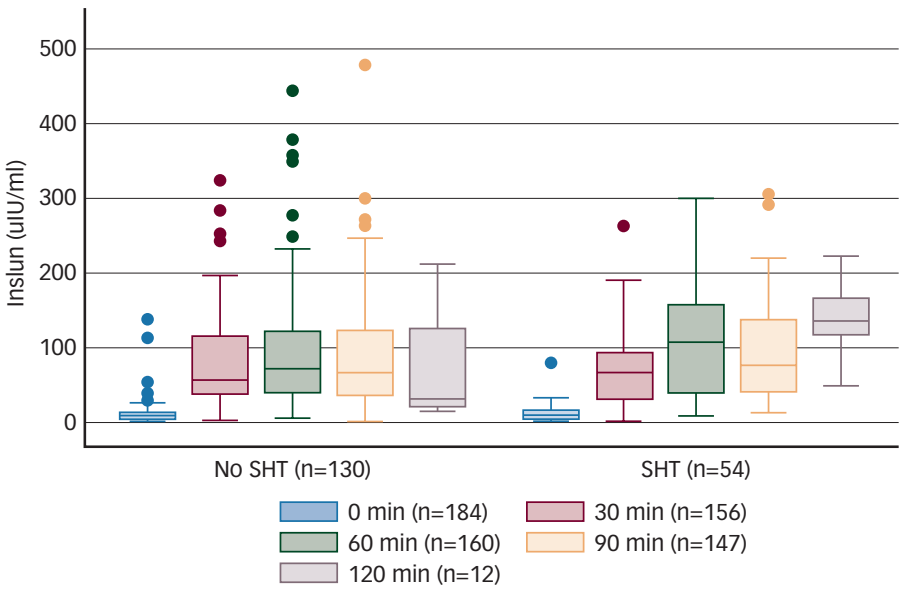

p>0.05; SHT= scalp hair thinning

Stepwise multivariable logistic regression analysis adjusting for age, smoking, and BMI was performed. Subjective hirsutism, oligomenorrhea, skipped meals, disturbed sleep, abnormal two-hour OGTT (IGT or DM), higher SHBG and lower LDL cholesterol levels emerged as independent positive predictors of SHT (Table 3). On adjusted analysis, oligomenorrhea and higher LDL levels were associated with significantly reduced likelihood of SHT. In addition, higher SHBG, skipped meals and abnormal two-hour OGTT were associated with an increased likelihood of SHT. In women with an abnormal two-hour OGTT, the likelihood of SHT was increased almost tenfold compared with those with normal glucose response following ingestion of $75 \mathrm{~g}$ glucose load. The final statistical model demonstrated $84 \%$ sensitivity for the outcome of SHT.

\section{Discussion}

We have used subjective SHT as a corollary for AA, a recognized, although less well-studied form of the hyperandrogenic symptomatology of PCOS. 5,18,20 
Prevalence of SHT symptom (27\%) acknowledged by our subjects with PCOS is comparable to rates reported by others. ${ }^{18}$ Women with PCOS were six times more likely to acknowledge SHT compared with the 6\% prevalence described in the general population of reproductive-aged females. ${ }^{4}$

While hyperandrogenemia has been hypothesized as a mechanism for SHT, existing data are equivocal. 5,18,20 Similarly, clinical hyperandrogenism has been inconsistently related to SHT in women with PCOS.5,18,20 Increased tissue levels of 5 alpha-reductase (the enzyme responsible for the conversion of testosterone to a more potent androgen dihydrotestosterone [DHT]) and DHT have been described in subjects with AA. ${ }^{23}$ Furthermore, sporadic evidence of women with SHT benefiting from the use of antiandrogens such as finasteride, (5 alpha-reductase inhibitor) has been reported. ${ }^{23,24}$ Although hirsute women were significantly more likely to acknowledge SHT in our population, these associations disappeared on adjusted analyses. Despite a higher prevalence of SHT in women with PCOS compared with the general population, we did not observe any relationship between excessive circulating androgen levels (ovarian or adrenal) and symptom of SHT, an observation previously recognized by others. ${ }^{18,21,22}$ We did not observe any relationship between acne and SHT, and this finding is also consistent with existing literature. ${ }^{5}$ Higher SHBG and lower LDL levels were observed in women complaining of SHT, phenomena that were previously reported by others. ${ }^{5}$ In a crosssectional study of Turkish women with PCOS, Ozdemir et al. had described significantly higher SHBG and significantly lower LDL levels in women with $\mathrm{AA}$ and these observations were recapitulated in our population. ${ }^{5}$ Women in the highest quartile of SHBG were three to four times more likely to report SHT compared with those with SHBG levels in the lowest quartile. Similar to observation by Ozdemir et al., women with SHT also exhibited significantly lower LDL, as well as significantly higher HDL:LDL ratios compared with those without this symptom. Thus, contrary to an assumption that systemic or focal androgen excess are relevant to the pathogenesis of AA, our data suggest that relative focal androgen and/ or estrogen deficiency (secondary to excess bound form of hormone) may be relevant to SHT in women with PCOS. Both LDL receptor and lipoprotein lipase mRNA expression are described in the skin appendages and a possible role for altered tissue steroidogenesis from cholesterol precursors as a mechanism for SHT can additionally be hypothesized. ${ }^{25}$

Hyperandrogenemia and insulin resistance, both hallmarks of PCOS, are recognized as contributory to risk for cardiovascular disease. ${ }^{20,26,27}$ Interestingly, insulin resistance and cardiovascular disease have also been linked to SHT in women. ${ }^{19,26-27}$ We did not observe any relationship between fasting or glucose-provoked insulin response and SHT in our population. Notably, however, we identified abnormal glucose homeostasis as a robust and independent predictor of SHT in women with PCOS. Although our study design does not allow any insight into mechanisms, end organ microvascular damage may be hypothesized as a possible mechanism for SHT given that vasculotoxicity and atherogenesis are well-recognized sequelae to chronic hyperglycemia. ${ }^{16}$ These observations raise the question of whether AA or SHT could be construed as an indicator of cardiovascular wellbeing in women with PCOS. Although less well-studied in women, hair loss and SHT has been linked to cardiovascular risk factors such as the presence of metabolic syndrome and insulin resistance..$^{26-27}$ The relationship between hair loss and cardiovascular disease is well described in men. Lesko et al. showed that the risk of myocardial

\section{Table 3: Predictors of scalp hair thinning}

\begin{tabular}{|c|c|c|}
\hline Parameters & $\begin{array}{l}\text { Unadjusted } \mathrm{OR}(95 \% \mathrm{Cl}) \\
\mathrm{P} \text { value }\end{array}$ & $\begin{array}{l}\text { Adjusted }{ }^{\mathrm{a}} \mathrm{OR}(95 \% \mathrm{Cl}) \\
\text { P value }\end{array}$ \\
\hline \multirow[t]{2}{*}{ Subjective hirsutism ${ }^{b}$} & $2.0(1.1-3.91)$ & $2.2(0.8-5.7)$ \\
\hline & 0.035 & 0.110 \\
\hline \multirow[t]{2}{*}{ Oligomenorrhea } & $0.6(0.32-1.32)$ & $0.3(0.1-0.9)$ \\
\hline & 0.230 & 0.031 \\
\hline \multirow[t]{2}{*}{ Disturbed sleep } & $1.9(1.1-3.4)$ & $1.3(0.6-3.2)$ \\
\hline & 0.026 & 0.470 \\
\hline \multirow[t]{2}{*}{ Skipped meals } & $2.3(1.2-4.2)$ & $3.1(1.3-67.3)$ \\
\hline & 0.008 & 0.017 \\
\hline \multirow[t]{2}{*}{ Asian race } & $3.9(1.6-9.5)$ & $3.5(0.78-16.6)$ \\
\hline & 0.003 & 0.094 \\
\hline \multirow[t]{2}{*}{ SHBG highest tertile ${ }^{c}$} & $2.05(1.05-3.98)$ & $3.4(1.2-9.8)$ \\
\hline & 0.034 & 0.020 \\
\hline \multirow[t]{2}{*}{ SHBG mid tertilec } & $1.7(0.87-3.32)$ & $2.8(1.0-7.8)$ \\
\hline & 0.120 & 0.056 \\
\hline \multirow[t]{2}{*}{ Abnormal 2h OGTT ${ }^{d}$} & $3.5(1.5-8.2)$ & $9.9(3.1-30.2)$ \\
\hline & 0.003 & $<0.001$ \\
\hline \multirow[t]{2}{*}{ LDL Cholesterol (mg/dl) } & $0.98(0.97-0.99)$ & $0.96(0.97-0.99)$ \\
\hline & 0.002 & 0.026 \\
\hline
\end{tabular}

Associations are presented as odds ratio $(95 \% \mathrm{Cl})$ : a adjustment variables included subjective hair excess, oligomenorrhea (defined as intermenstrual duration $>35$ days), histories of disturbed sleep and erratic eating /skipped meals, Asian versus other race, SHBG, abnormal glucose response during OGTT and LDL cholesterol level. ' Subjective reporting of excess facial and body hair on $\mathrm{MFG}$ scale; $M F G>8$ creferent category is of lowest tertile dabnormal 2 h OGT included evidence of either IGT or DM based on specified thresholds of glucose levels. $D M=$ diabetes mellitus; $L D L=10 \mathrm{w}$-density lipoprotein; OGTT= oral $75 \mathrm{~g}$ glucose tolerance test; $S H B G=$ sex hormone binding globulin; SHT = scalp hair thinning

\section{Figure 3: Higher prevalence of aberrations in glucose homeostasis noted in women acknowledging scalp hair thinning}
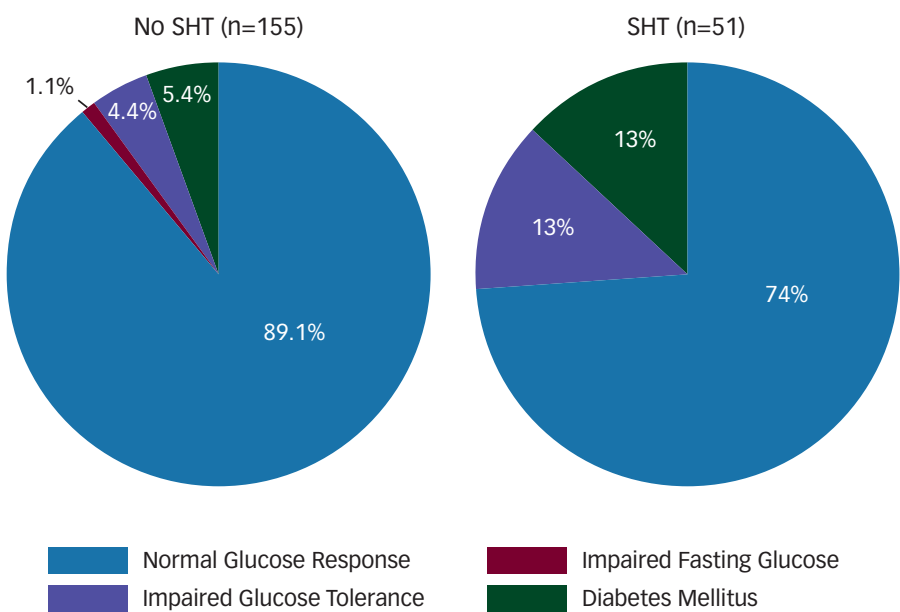

$p=0.054$ (corrected for multiple comparisons between groups). SHT= scalp hair thinning

infarction was directly correlated to the presence and severity of vertex baldness. ${ }^{28}$ Abnormalities in glucose homeostasis are recognized as being of pathogenic relevance to peripheral vascular disease and end organ damage such as neuropathy and nephropathy. Our data identify scalp hair as a target tissue that is compromised by abnormal glucose homeostasis. Psychological burden relating to hair loss is well-recognized both in men and women and a high prevalence of depressive symptoms is also described, although inconsistently, in association with AA. ${ }^{29}$ The proportion 
of women acknowledging a history of depression was comparable between those with and without SHT (Table 1); prevalence of anxiety was higher in those with SHT, but the difference was not of statistical significance. Overall, these findings are consistent with existing data. ${ }^{18}$ Notably, women with SHT were significantly more likely to acknowledge disturbed sleep, a symptom that may reflect psychological unrest, and this relationship held on adjusted analyses (Table 3).

Limitations of our study include a retrospective approach, cross-sectional study design, the absence of a control group of women without PCOS and the relatively small sample size comprised of subjects attending a single specialist's clinical practice. Although subjectivity of complaint of SHT is also a limitation, one cannot minimize the relevance of an individual's symptom-related subjective distress; hence, subjectivity of symptom may even be argued as a strength of this endeavor. While none of the subjects were taking antiandrogen therapy at the time of evaluation, information on the use of topical therapy (such as minoxidil) is not available. Not only did circulating androgen levels not relate to SHT, but, intriguingly, higher SHBG levels predicted an increased likelihood for SHT; lower scalp tissue exposure to free androgens and estrogen can be hypothesized as a mechanism that could underlie the observed associations, although again the study design limits our ability to interpret underlying mechanisms. Similarly, mechanisms whereby oligomenorrhea could be protective against SHT are unclear, although relative estrogen excess in the setting of anovulatory cycles can be suggested as a plausible mechanism.

\section{Conclusions}

SHT is a prevalent symptom in women with PCOS. Tissue, rather than systemic endocrine milieu appears to be of relevance for scalp hair density and growth. Impaired glucose homeostasis is identified as a relevant and potentially modifiable predictor of SHT in this population. Given the prevalence, the psychological, and now apparent metabolic corollaries to SHT, future studies must focus on pathogenic mechanisms contributory to this common entity.
1. Zawadski JK, Dunaif A, Diagnostic criteria for polycystic ovarian syndrome, towards a rational approach. In: Dunaif A, Given JR, Haseltine F, Merriam G (eds), Polycystic ovary syndrome, Boston: Blackwell Scientific 1992:377-884.

2. Rotterdam ESHRE/ASRM-Sponsored PCOS Consensus Workshop Group, Revised 2003 consensus on diagnostic criteria and longterm health risks related to polycystic ovary syndrome, Fertil Steril, 2004;81:19-25

3. Knochenhauer ES, Key TJ, Kahsar-Miller M, et al., Prevalence of the polycystic ovary syndrome in unselected black and white women of the southeastern United States: a prospective study, I Clin Endocrinol Metab, 1998:83:3078-82.

4. Birch MP, Messenger IE, Messenger AG, Hair density, hair diameter and the prevalence of female pattern hair loss, Br J Dermatol, 2001:144:297-304.

5. Ozdemir S, Ozdemir M, Gorkemli H, et al., Specific dermatologic features of the polycystic ovary syndrome and its association with biochemical markers of the metabolic syndrome and hyperandrogenism, Acta Obstet Gynecol Scand, 2010;89:199-204

6. Carmina E, Rosato F, Janni A, et al., Relative prevalence of different androgen excess disorders in 950 women referred because of clinical hyperandrogenism, $J$ Clin Endocrinol Metab 2006;91:2-6.

7. Hassa H, Tanir HM, Yildiz Z, Comparison of clinical and laboratory characteristics of cases with polycystic ovarian syndrome based on Rotterdam's criteria and women whose only clinical signs are oligo/anovulation or hirsutism, Arch Gynecol Obstet, 2006:274:227-32.

8. Van Der Donk J, Hunfeld JAM, Passchier J, et al., Quality of life and maladjustment associated with hair loss in women with alopecia androgenetica, Soc Sci Med, 1994;38:159-69.
9. Shapiro J, Hair loss in women, N Eng/ J Med, 2007;357:1620-30.

10. Norwood OT, Lehr B, Female androgenetic alopecia: a separate entity, Dermatol Surg, 2000; 26:679-82.

11. Cinar N, Kizilarslanoglu MC, Harmanci A, et al., Depression, anxiety and cardiometabolic risk in polycystic ovary syndrome, Hum Reprod, 2011:26:3339-45.

12. Cash TF, Price VH, Savin RC, Psychological effects of androgenetic alopecia on women: comparison with balding men and with female control subjects, J Am Acad Dermatol, 1993:29:568-75.

13. Practice bulletin no. 128: Diagnosis of Abnormal Uterine Bleeding in Reproductive-aged Women, Obstet Gynecol, 2012:128:197-206.

14. Spitzer RL, Kroenke K, Williams JB, et al., Validation and Utility of a Self-report of PRIME-MD, The PHQ primary care study, JAMA 1999; 282:1737-44

15. Hatch R, Rosenfield RL, Kim MH, Tredway D, Hirsutism: Implications, etiology and management, Am J Obstet Gynecol, 1981:140:815-30

16. American Diabetes Association, Diagnosis and Classification of Diabetes Mellitus, Diabetes Care, 2014;37(Suppl. 1):S81-S90.

17. Matthews DR, Hosker JP Rudenski AS, et al. Homeostasis mode assessment: insulin resistance and fl-cell function from fasting plasma glucose and insulin concentrations in man, Diabetologia 1985;28:412-9

18. Quinn M, Shinkai K, Pasch L, et al., Prevalence of androgenic alopecia in subjects with polycystic ovary syndrome and characterization of associated clinical and biochemical features, Fertil Steril, 2014:101:1129-34.

19. Rotterdam ESHRE/ASRM-Sponsored PCOS consensus workshop group, Revised 2003 consensus on diagnostic criteria and long term health risks related to polycystic ovary syndrome, Human
Reproduction, 2004;19:41-7.

20. Cela E, Robertson C, Rush K, et al., Prevalence of polycystic ovaries in women with androgenic alopecia, Eur J Endocrinol, 2003; 149:439-42.

21. Kaufman K, Androgens and alopecia, Mol Cel Endocr. 2002;198:89-95.

22. Emer JJ, Levy LL, Female pattern alopecia: current perspectives, Int J Women's Health, 2013;5:541-56.

23. Price VH, Androgenetic alopecia in women, I Invest Dermatol symp Proc, 2003:8:24-7.

24. Shum KW, Cullen DR, Messenger AG, Hair loss in women with hyperandrogensism: Four cases responding to finasteride, J Am Acad Dermatol, 2002:47:733-9.

25. Smythe CD, Greenall M. Kealey T, The activity of HMG-COA reductase and acetyl- CoA carboxylase in human apocrine sweat glands, sebaceous glands and hair follicles is regulated by phosphorylation and by exogenous cholesterol, J Invest Dermatol, 1998;111:139-48.

26. Matilenin V, Laakso M, Hirsso $P$, et al., Hair loss, insulin resistance and heredity in the middle aged woman. A population based study, Eur I Prev Cardiol, 2003:10:227-31.

27. Arias-Santiago S, Guiterrez-Salmeron MT, Castellote-Caballero $L$, et al., Androgenetic alopecia and cardiovascular risk factors in men and women: A comparative study, J Am Acad Dermatol, 2010;63:420-9.

28. Lesko SM, Rosenberg L, Shapiro S, A Case-Control Study of Baldness in Relation to Myocardial Infarction in Men, JAMA 1993:269:998-1003.

29. Tabolli S1, Sampogna F, di Pietro C, et al., Health status, coping strategies, and alexithymia in subjects with androgenetic alopecia: a questionnaire study, Am I Clin Dermatol 2013;14:139-45. 